\begin{tabular}{|c|l|}
\hline Title & Genetic diversity within the Japanese badgers (Meles anakuma), as reveal ed by microsatellite analysis \\
\hline Author(s) & Tashima, Sara; Kaneko, Yayoi; A nezaki, Tomoko; Baba, Minoru; Y achimori, Shuuji; Masuda, Ryuichi \\
\hline Citation & $\begin{array}{l}\text { Mammal Study, 35(4), 221-226 } \\
\text { https://doi.org/10.3106/041.035.0401 }\end{array}$ \\
\hline Issue Date & 2010-12 \\
\hline Doc URL & http://hdl.handle.net/2115/47533 \\
\hline Type & article \\
\hline File Information & MS35-4_221-226.pdf \\
\hline
\end{tabular}

Instructions for use 


\title{
Genetic diversity within the Japanese badgers (Meles anakuma), as revealed by microsatellite analysis
}

\author{
Sara Tashima ${ }^{1}$, Yayoi Kaneko ${ }^{2}$, Tomoko Anezaki ${ }^{3}$, Minoru Baba ${ }^{4}$, Shuuji Yachimori ${ }^{5}$ and \\ Ryuichi Masuda ${ }^{1, *}$ \\ ${ }^{1}$ Department of Natural History Sciences, Graduate School of Science, Hokkaido University, Sapporo 060-0810, Japan \\ ${ }^{2}$ Wildlife Conservation Laboratory, Division of Ecosciences, Tokyo University of Agriculture and Technology, Fuchu \\ 183-8509, Japan \\ ${ }^{3}$ Gunma Museum Natural History, Tomioka 370-2345, Japan \\ ${ }^{4}$ Kitakyushu Museum of Natural History and Human History, Kitakyushu 805-0071, Japan \\ ${ }^{5}$ Shikoku Institute of Natural History, Susaki 785-0023, Japan
}

\begin{abstract}
To further understand the population structures of the Japanese badgers (Meles anakuma) on the Japanese islands, we analyzed their bi-parentally inherited microsatellites. Based on genotypes of nine microsatellite loci, the badgers were divided into five discrete clusters: three clusters from the Honshu Island, one from Kyushu and one from Shikoku. We propose that this genetic differentiation among badgers from the Honshu, Shikoku and Kyushu Islands is as a consequence of geographical isolation caused by the Seto Inland Sea. Furthermore, the cluster containing individuals from Shikoku was more differentiated from the other clusters, plausibly attributable to the earlier geological separation of the Shikoku Island from the Honshu and Kyushu Islands. The three clusters in Honshu, however, did not correspond precisely with geographical locations. As indicated in previous studies, based on mitochondrial DNA analysis, the genetic relationships within the Japanese badgers might reflect recent population expansion, occurring over a relatively short evolutionary time-scale. The findings preliminarily indicate that the Japanese badgers do not possess the high levels of philopatry seen in the European badger (Meles meles), a closely related species, although further analyses using balanced sample sizes from a wider range is required.
\end{abstract}

Key words: diversity, geographic barrier, Japanese badger, Meles anakuma, microsatellite.

The Japanese badger (Meles anakuma) is a mediumsized mustelid endemic to Japanese islands. Meles anakuma was thought to be a subspecies of the Eurasian badger (M. meles); this former species extending across the Palaearctic, from the Japanese islands to the United Kingdom and Ireland through the mid-latitude Eurasian Continent. Three subspecies groups were recognized: M. m. meles, M. m. arenarius-leptorhyncus and M. $m$. amurensis-anakuma (Heptner et al. 1967). Recently, however, these subspecies have been promoted to independent species status, the European badger (M. meles), the Asian badger (M. leucurus), and the Japanese badger (M. anakuma), based on morphological features such as mask coloration (Abramov 2003), baculum structure (Abramov 2002) and unique parasitic fleas (Abramov and Medvedev 2003). Moreover, phylogenetic analyses of mitochondrial DNA (mtDNA) (Kurose et al. 2001; Marmi et al. 2006) and nuclear DNA (Sato et al. 2003) have demonstrated the large differentiation between Japanese and continental badgers. Kurose et al. (2001) reported the low mtDNA cytochrome $b$ genetic diversity within Japanese badgers, and found no relationships between genetic distances and the geographic distances between sampling locations.

Japanese badgers are distributed in the Honshu, Shikoku and Kyushu Islands, inhabiting various environments such as deciduous larch plantation, deciduous forests, cedar/cypress plantation, agriculture land, inter alia (see Kaneko 2009). The badger is currently treated as a game species in Japan although the numbers hunted

*To whom correspondence should be addressed. E-mail: masudary@ees.hokudai.ac.jp 
per year have declined from 7,000 individuals in the 1970 s to less than 2,000 in the late 1980s, Kaneko (2009) proposing this decline to be as a result of loss of interest in badgers as game animals, rather than intrinsic population decline. Although this species is listed as 'least concern' by the Japanese Ministry of the Environment, a $7 \%$ reduction in its distribution range occurred between 1978 and 2003 (Ministry of Environment, Japan 2004) and 11 out of 46 prefectures list the Japanese badger in the red data book (Kaneko 2009). Further survey work is imperative to assess the species' national status in Japan.

To further understand the genetic population structures of the Japanese badgers, here we investigated variations between badgers from various regions in biparentally inherited and allele frequencies. These genetic data are then used to construct a framework for differentiation within the Japanese badgers.

\section{Materials and methods}

\section{Samples and DNA extraction}

Tissue samples, hairs and bloods were obtained from 85 Japanese badgers, which were roadkilled, hunted, and captured at ecological studies between 1990 and 2009. Muscle, liver and skin tissues were preserved in ethanol, hairs were dried at room temperature, and bloods were frozen at $-80^{\circ} \mathrm{C}$ until use. Sampling locations and numbers are shown in Figure 1. DNA extraction was per-

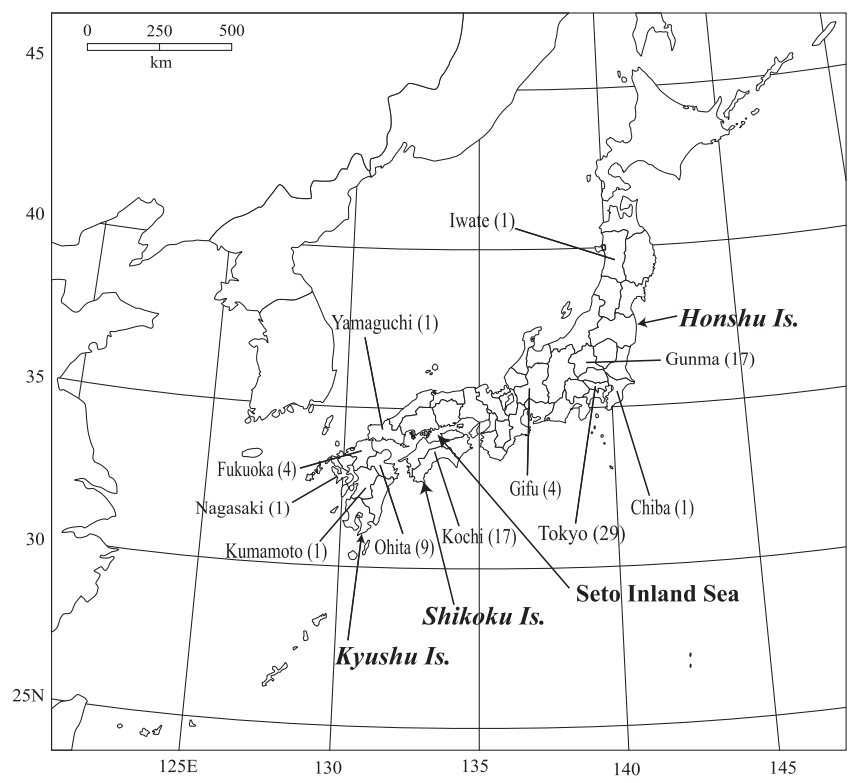

Fig. 1. The sampling locations of Meles anakuma on the Japanese islands. Numbers in parentheses indicate the numbers of individuals sampled from these locations. Lines within main islands of Japan show prefectures' boundaries. formed by using QiAamp DNA Micro Kit (Qiagen) for hair roots and DNeasy Blood \& Tissue Kit (Qiagen) for the other tissues. Extracted DNA samples were dissolved in $200 \mu \mathrm{l}$ of $10 \mathrm{mM}$ Tris/1 mM EDTA/pH 8.0 (TE) buffer, and preserved at $4{ }^{\circ} \mathrm{C}$.

\section{Microsatellites analysis}

Nine polymorphic microsatellite loci were amplified with the suite of primers previously reported by Carpenter et al. (2003) (Mel101, Mel102, Mel104-Mel110). An aliquot $(10 \mu \mathrm{l})$ of polymerase chain reaction $(\mathrm{PCR})$ solution was comprised by $1 \mu \mathrm{l}$ of $10 \times$ PCR buffer, $0.8 \mu \mathrm{l}$ of $2.5 \mathrm{mM}$ dNTP mixture, $0.1 \mu \mathrm{l}$ of TaqDNA polymerase ( 5 units/ $\mu 1$, Takara), $0.3 \mu$ l of above each primer and $1 \mu \mathrm{l}$ of extracted DNA, and $6.8 \mu \mathrm{l}$ of distilled water. The PCR amplifications were carried out in a PCR thermal cycler (Takara TP600), one cycle of $94^{\circ} \mathrm{C}$ for $3 \mathrm{~min}$ and 35 cycles of $94^{\circ} \mathrm{C}$ for $15 \mathrm{sec} ; 54^{\circ} \mathrm{C}$ for $20 \mathrm{sec} ; 72^{\circ} \mathrm{C}$ for $30 \mathrm{sec}$; followed by one cycle of $72^{\circ} \mathrm{C}$ for $10 \mathrm{~min}$. These PCR products were then processed in an automated DNA sequencer (Hitachi SQ5500), and resultant data were analyzed using FRAGLYS v. 2.0 software (Hitachi).

Observed $\left(H_{\mathrm{O}}\right)$ and expected $\left(H_{\mathrm{E}}\right)$ heterozygosities, mean numbers of alleles per locus and value of pairwise $F_{\text {ST }}$ were calculated using ARLEQUIN 3.1 software (Excoffier et al. 2005). Departures from Hardy-Weinberg equilibrium and linkage equilibrium were tested for each of the nine loci using GENEPOP 3.4 software (Raymond and Rousset 1995) applying the Markov chain method according to the algorithm of Guo and Thompson (1992). Based on values of pairwise $F_{\mathrm{ST}}$, a neighbor-joining tree (Saitou and Nei 1987) was constructed using MEGA 4 software (Tamura et al. 2007). The most parsimonious number of populations was estimated using STRUCTURE 2.2 software (Pritchard et al. 2000). This program characterizes each population by allele frequencies at each locus. Ten replicates were performed in order to establish each value of $K$, where $K$ represents the optimal number of genetic clusters (i.e. populations), testing a range of $K$ from 3 to 10 (with 100,000 burnin and 100,000 Markov Chain Monte Carlo replicates after burnin). Individuals with greater than a $50 \%$ assignment rate in favor of one population were assumed to belong to that population. In addition, Nei's standard genetic distance $\left(D_{\mathrm{S}}\right)$ (Nei 1978) was calculated for each subpopulation using SPAGeDi 1.2 software (Hardy and Vekemans 2002) and the phylogenetic tree based on the neighbor-joining method (Saitou and Nei 1987) was constructed based on $D_{\mathrm{s}}$ by using MEGA 4 software. 
Table 1. Diversity of nine microsatellite loci of seven badger populations

\begin{tabular}{lcccc}
\hline \multirow{2}{*}{$\begin{array}{c}\text { Regional } \\
\text { population* }\end{array}$} & $\begin{array}{c}\text { No. of } \\
\text { animals }\end{array}$ & Alleles/locus & \multicolumn{2}{c}{ Heterozygosities } \\
\cline { 4 - 5 } & & & $\begin{array}{c}\text { Observed } \\
\left(H_{\mathrm{O}}\right)\end{array}$ & $\begin{array}{c}\text { Observed } \\
\left(H_{\mathrm{E}}\right)\end{array}$ \\
\hline Iwate & 1 & 1.4 & - & - \\
Kanto & 47 & 7.9 & 0.454 & 0.638 \\
Gifu & 4 & 3.4 & 0.389 & 0.607 \\
Yamaguchi & 1 & 1.4 & - & - \\
Shikoku & 17 & 4.8 & 0.484 & 0.529 \\
Kyushu & 15 & 4.8 & 0.444 & 0.530 \\
\hline
\end{tabular}

* Kanto consists of individuals from Tokyo $(n=29)$, Chiba $(n=1)$ and Gunma $(n=17)$. Shikoku consists of individuals from Kochi $(n=17)$. Kyushu consists of individuals from Fukuoka $(n=4)$, Nagasaki $(n=1)$, Oita $(n=9)$ and Kumamoto $(n=1)$.

\section{Results}

\section{Genetic diversity of Japanese badgers}

Nine microsatellite loci in 85 Japanese badgers were genotyped. As only a single individual was genotyped in each of Iwate and Yamaguchi Prefectures, these data were excluded from heterozygosity calculation in Table 1. The Kanto population was comprised by individuals from Tokyo $(n=29)$, Chiba $(n=1)$ and Gunma $(n=17)$ Prefectures. The Gifu population consisted of four individuals from Gifu Prefecture. The Shikoku population consisted of individuals $(n=17)$ from Kochi Prefecture. The Kyushu population consisted of individuals from Fukuoka $(n=4)$, Nagasaki $(n=1)$, Oita $(n=9)$ and Kumamoto $(n=1)$ Prefectures.

The number of alleles measured ranged from three (at Mel108) to 24 (at Mel105). The mean number of alleles per locus over all populations was 4.0 and the highest number was 7.9, from the Kanto population. Observed heterozygosity $\left(H_{\mathrm{O}}\right)$ ranged from 0.389 to 0.484 , and the expected heterozygosity $\left(H_{\mathrm{E}}\right)$ ranged from 0.529 to 0.638 (Table 1). The value (1.4) of alleles per locus for both single individuals from Iwate and Yamaguchi Prefectures (Table 1) indicated multiple loci in each of the two badgers were homozygous.

The neighbor-joining tree based on $F_{\mathrm{ST}}$ values showed that the Shikoku population is more distant from the other populations (Fig. 2). The highest value of pairwise $F_{\text {ST }}(0.235)$ occurred between the Shikoku and Kyushu populations. The lowest $(0.104)$ occurred between Kanto and Gifu populations.

\section{Population structures of Japanese badgers}

The STRUCTURE analysis showed that the highest

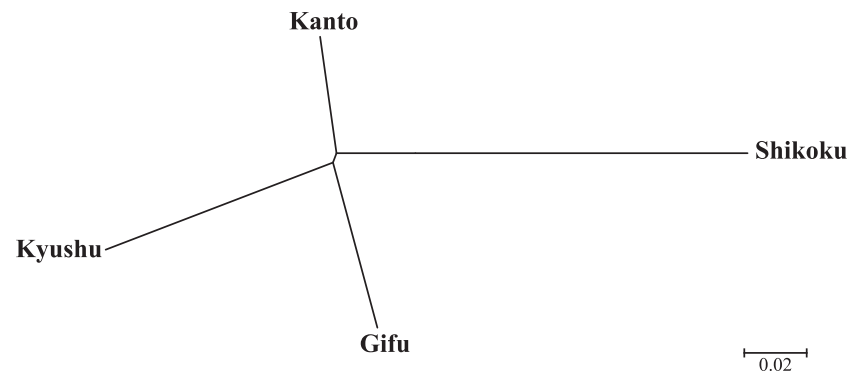

Fig. 2. The neighbor-joining tree based on pairwise $F_{\mathrm{ST}}$ between regional populations of Meles anakuma, obtained by microsatellite analysis. The scale below shows $F_{\mathrm{ST}}$ values.

log-likelihood value was found at $K=5$, consequently the most optimal number of populations of the Japanese badgers obtained in the present study was established to be five, referenced as clusters I-V. Of the 85 individuals genotyped, 81 were assigned to either of these five clusters with $>50 \%$ assignment rates. Bayesian clusterings approximated with the populations which we defined on the basis of regions. Out of the five clusters, three clusters I, II and III were shared by the majority of individuals from the Kanto population. The Shikoku and Kyushu populations were assigned to cluster IV and V with $>91 \%$ and $>85 \%$ assignment rates, respectively (Table 2). Three individuals from Gifu, located in central Honshu, were assigned to cluster IV or V, and not to any of clusters I, II and III distributed in Kanto. The neighbor-joining tree based on $D_{\mathrm{S}}$ values showed a broader differentiation of cluster IV from the other clusters (Fig. 3).

\section{Discussion}

\section{Genetic relationships among the Japanese badgers}

Regional populations defined by sampling locations corresponded well with genetic populations inferred by microsatellite genotyping (Table 2). Bayesian clusterings, established by STRUCTURE analysis, revealed that the Shikoku and Kyushu populations were separated from each other and from most individuals from the Honshu Island: that is, cluster IV consisted of all 17 individuals from Shikoku and one from Gifu, and cluster V consisted of all 15 individuals from Kyushu and two from Gifu (Table 2).

Tsuchiya et al. (2000) reported that the Shikoku and Kyushu populations of the large Japanese mole (Mogera wogura) are also separated from one another, as well as from Honshu populations, based on mtDNA cytochrome 
Table 2. Assignment of the Japanese badgers to five clusters

\begin{tabular}{|c|c|c|c|c|c|c|c|c|c|c|c|}
\hline \multirow{3}{*}{ Clusters* } & \multicolumn{11}{|c|}{ Regional populations } \\
\hline & \multirow{2}{*}{ Iwate } & \multicolumn{3}{|c|}{ Kanto } & \multirow{2}{*}{ Gifu } & \multirow{2}{*}{ Yamaguchi } & \multirow{2}{*}{$\begin{array}{c}\text { Shikoku } \\
\text { Kochi }\end{array}$} & \multicolumn{4}{|c|}{ Kyushu } \\
\hline & & Tokyo & Gunma & Chiba & & & & Fukuoka & Nagasaki & Oita & Kumamoto \\
\hline I & 1 & 16 & 1 & & & & & & & & \\
\hline II & & 9 & 3 & 1 & & & & & & & \\
\hline III & & 3 & 12 & & & & & & & & \\
\hline VI & & & & & 1 & & 17 & & & & \\
\hline $\mathrm{V}$ & & & & & 2 & & & 4 & 1 & 9 & 1 \\
\hline Non-assigned individuals & 0 & 1 & 1 & 0 & 1 & 1 & 0 & 0 & 0 & 0 & 0 \\
\hline
\end{tabular}

* Defined by STRUCTURE test using genotypes.

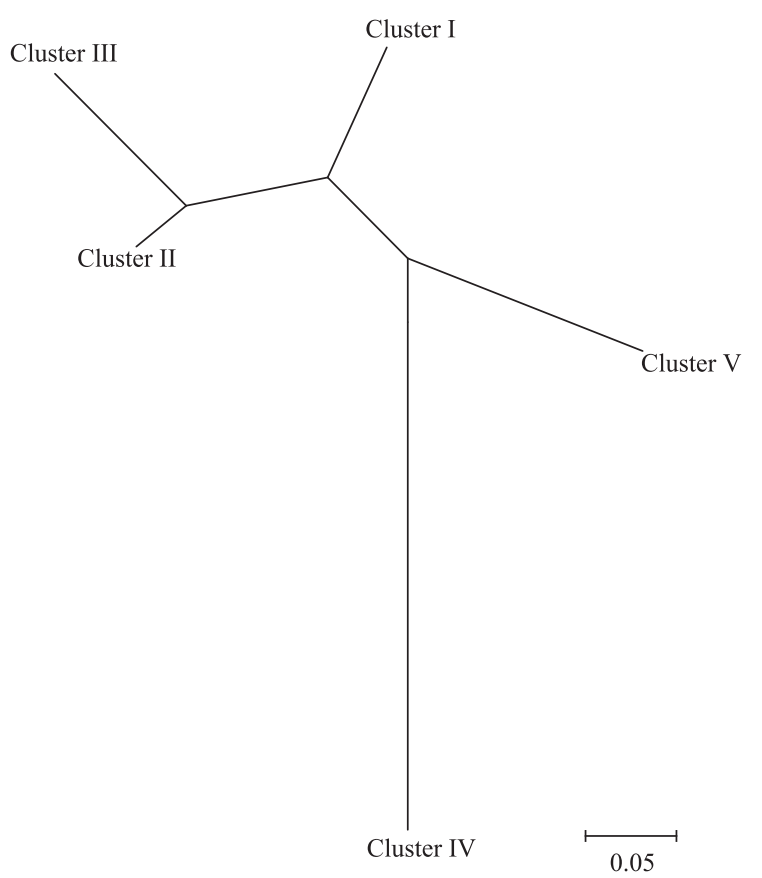

Fig. 3. The neighbor-joining tree based on pairwise $D_{\mathrm{s}}$ between Meles anakuma clusters inferred by the STRUCTURE analysis. The scale below shows $D_{\mathrm{s}}$ values.

$b$ data. The Seto Inland Sea (see Fig. 1), which presently separates the Honshu, Shikoku and Kyushu Islands, formed about 5,000 years ago (Ohshima 1990). Consequently it is highly plausible that the differentiation of the Shikoku and Kyushu populations from the Kanto and Gifu populations in the Honshu Island might have arisen as a result of the geographical isolation caused by the formation of the Seto Inland Sea. For the Japanese sika deer (Cervus nippon), however, the Shikoku Island is a sympatric region of the northern and southern mtDNA lineages (Yamada et al. 2006). In the present study, the individuals from Gifu at central Honshu were included in the clusters of Shikoku and Kyushu. Further investigation is required to examine the full ramifications of the ontogeny of the Seto Inland Sea on the extended population structures of the Japanese badgers, using more samples from central and western Honshu.

The present study demonstrates that the Shikoku population is the most differentiated (Fig. 2). The genetic separation of the Shikoku population could have been affected by formation of the Seto Inland Sea. During the formation process, the Shikoku and Honshu Islands first became separated about 7,000 years ago, with the Kyushu Island becoming separated from Honshu around about 5,000 years ago (Ohshima 1990). The earlier separation of Shikoku from the other main islands and consequent isolation, would support our observation of Shikoku having the most genetically differentiated population.

\section{Genetic structures within the Kanto and Gifu populations}

For the badgers from the Honshu Island, the Kanto populations consisted of 29 individuals from Tokyo, one from Chiba and 17 from Gunma Prefectures; the Gifu population consisted of four from Gifu Prefecture; and one badger from Iwate Prefecture and one badger from Yamaguchi Prefecture were examined. The STRUCTURE analysis showed that the badgers from the Honshu Island were divided into three genetic clusters. Most animals grouped within each of the three genetic clusters in Honshu were distributed in geographically proximate areas. For example, cluster III consisted of 12 individuals from Gunma Prefecture, but it also included three individuals from Tokyo. This genetic population structure could be explained by the recent population expansion. Based on the mtDNA analysis, Kurose et al. (2001) reported that the current distribution of the Japanese 
badgers had been formed through the repeated 'increase and reduction' of the total population size, with concomitant changes in distribution range due to the palaeoenvironmental changes, such as glacial-interglacial episodes.

In addition, Kurose et al. (2001) also suggested the possibility that the recent expansion of the Japanese badgers may have resulted in their unestablished geographical structures. In accord with the mtDNA data, the present study of microsatellites also did not identify any explicit regional genetic structure in the Japanese badgers of Honshu. By contrast, microsatellite analysis of the European badger (Meles meles) (Pope et al. 2006, 2007), a species closely related to the Japanese badger, established a clear population structure, which has been attributed to the high level of natal philopatry observed in study areas in the British Isles (Kruuk and Parish 1982; Cheeseman et al. 1987; Macdonald et al. 2008). If the Japanese badger were to observe strict natal philopatry, then resultant genetic population structures should be concordant with those described for the European badger (Pope et al. 2006, 2007): this, however, was not consistent with our findings among the Japanese badgers of Honshu, which did not show clear genetic structures. This indicates that the level of the natal philopatry of the Japanese badger may not be as high as that of the European badger. Moreover, in contrast to the European badger, the ecological traits of the Japanese badgers are not well known (Yamamoto 1986, 1991; Kaneko 2001, 2002; Tanaka et al. 2002). For another reason, the small number of sampling points and unbalanced sample sizes in the present study might have partly led to the resultant unestablished geographical structures of the Japanese badgers. To further understand the evolutionary ecology, breeding systems and life-history traits of the Japanese badgers, not only will genetic analyses using samples collected from a wider range be required, but also further ecological research into fundamental biology.

Acknowledgments: We thank Dr. Tsunenori Tsujimoto (Morioka Zoological Park), Dr. Hiroshi Tanaka (Yamaguchi Museum), and Mr. Seiichiro Dakemoto (Takayama City) for supplying badger samples. We also thank Dr. Chris Newman (University of Oxford) and Dr. Nigel Tantram for their help of language. This study was supported in part by a Grant-in-Aid for Scientific Research (No. 17405012) from the Japan Society for the Promotion of Science.

\section{References}

Abramov, A. V. 2002. Variation of the baculum structure of the Palearctic badger (Carnivora, Mustelidae, Meles). Russian Journal of Theriology 1: 57-60.

Abramov, A. V. 2003. The head colour pattern of the Eurasian badgers (Mustelidae, Meles). Small Carnivore Conservation 29: 5-7.

Abramov, A. V. and Medvedev, S. G. 2003. Notes on zoogeogtaphy and taxonomy of the badgers (Carnivore: Mustelidae: Meles) and some of their fleas (Siphonaptera: Ceratophylidae: Paraceras). Zoosystematica Rossica 11: 398-402.

Carpenter, P. J., Dawson, D. A., Greig, C., Parham, A., Cheeseman, C. L. and Burke, T. 2003. Isolation of 39 polymorphic microsatellite loci and the development of a fluorescently labeled marker set for the Eurasian badger (Meles meles) (Carnivora: Musutelidae). Molecular Ecology Notes 3: 610-615.

Cheeseman, C. L., Wilesmith, J. W., Ryan, J. and Mallinson, P. J. 1987. Badger population dynamics in a high-density area. Symposia of the Zoological Society of London 58: 279-294.

Excoffier, L., Laval, G. and Schneider, S. 2005. Arlequin ver. 3.0: an integrated software package for population genetics data analysis. Evolutionary Bioinformatics Online 1: 47-50.

Guo, S. W. and Thompson, E. A. 1992. Performing the exact test of Hardy-Weinberg proportion for multiple allels. Biometrics 48: 361-372.

Hardy, O. and Vekemans, X. 2002. SPAGeDi: a versatile computer program to analyse spatial genetic structure at the individual or population level. Molecular Ecology Notes 2: 618-620.

Heptner, V. G., Naumov, N. P., Yutgenson, P. B., Sludskii, A. A., Chirkova, A. F. and Bannikov, A. G. 1967. Subfamily Melinae, Genus Meles Brisoon, 1762, Meles meles (Linnaeus, 1758). In (V. G. Heptner and N. P. Naumov, eds.) Mammals of the Soviet Union, Vol. II, Part 1 Sirenia and Carnivora, pp. 1228-1282. Vysshaya Shkola, Moscow (Scientific Publisher, Enfield, for the English-translated version).

Kaneko, Y. 2001. Life cycle of the Japanese badger (Meles meles anakuma) in Hinode Town, Tokyo. Mammalian Science [Honyurui Kagaku] 41: 53-64 (in Japanese with English summary).

Kaneko, Y. 2002. Inner structure of badger home range in Hinodetown. Japanese Journal of Ecology 52: 243-252.

Kaneko, Y. 2009. Meles anakuma Temminck, 1844. In (S. D Ohdachi, Y. Ishibashi, M. A. Iwasa and T. Saitoh, eds.) The Wild Mammals of Japan, pp. 258-260. Shoukadoh, Kyoto.

Kruuk, H. and Parish, T. 1982. Factors affecting population density, groups and territory size of the European badger Meles meles. Journal of Zoology 196: 31-39.

Kurose, N., Kaneko, Y., Abramov, A. V., Siriaroonrat, B. and Masuda, R. 2001. Low genetic diversity in Japanese populations of the Eurasian badger Meles meles (Mustelidae, Carnivora) revealed by mitochondrial cytochrome $b$ gene sequences. Zoological Science 18: 1145-1151.

Macdonald, D. W., Newman, C., Buesching, C. D. and Johnson, P. J. 2008. Male-biased movement in a high density population of the Eurasian badger (Meles meles). Journal of Mammology 89: 1077-1086.

Marmi, J., López-Giráldez, F., Macdonald, D. W., Calafell, F., Zholnerovskaya, E. and Domingo-Roura, X. 2006. Mitochondrial DNA reveals a strong phylogeographic structure in the badger across Eurasia. Molecular Ecology 15: 1007-1020.

Ministry of Environment, Japan. 2004. The sixth National Survey on the Natural Environment Report of the Distribution Survey of Japanese Animals (Mammals). Japan Wildlife Research Center, 213 pp. (in Japanese). 
Nei, M. 1978. Estimation of average heterozygosity and genetic distance from a small number of individuals. Genetics 89: 583-590.

Ohshima, K. 1990. The history of straits around the Japanese islands in the late-Quaternary. Quaternary Research 29: 193-208 (in Japanese with English abstract).

Pope, L. C., Domingo-Roura, X., Erven, K. and Burke, T. 2006. Isolation by distance and gene flow in the Eurasian badger (Meles meles) at both a local and broad scale. Molecular Ecology 15: 371-386.

Pope, L. C., Butlin, R. K., Wilson, G. J., Woodroffe, R., Erven, K., Conyers, C. M., Franklin, T., Delahay, R. Cheeseman, C. L. and Burke, T. 2007. Genetic evidence that culling increases badger movement: implications for the spread of bovine tuberculosis. Molecular Ecology 16: 4919-4929.

Pritchard, J., Stephens, M. and Donnelly, P. 2000. Inference of population structure using multilocus genotype data. Genetics 155: 945-959.

Raymond, M. and Rousset, F. 1995. GENEPOP (version 1.2): population genetics software for exact tests and ecumenicism. Journal of Heredity 86: 248-249.

Saitou, N. and Nei, M. 1987. The neighbor-joining method: a new method for reconstructing phylogenetic trees. Molecular Biology and Evolution 4: 406-425.

Sato, J. J., Hosoda, T., Wolsan, M., Tsuchiya, K., Yamamoto, M. and Suzuki, H. 2003. Phylogenetis relationships and divergence times among mustelids (Mammalia: Carnivora) based on nucleotide sequences of the nuclear interphotoreceptor retinoid binding protein and mitochondrial cytochrome $b$ genes. Zoological Science 20: 243-264

Tamura, K., Dudley, J., Nei, M. and Kumar, S. 2007. MEGA4: Molecular evolutionary genetics analysis (MEGA) software version 4.0. Molecular Biology and Evolution 24: 1596-1599.

Tanaka, H., Yamanaka, A. and Endo, K. 2002. Spatial distribution and sett use by the Japanese badger, Meles meles anakuma. Mammal Study 27: 15-22

Tsuchiya, K., Suzuki, H., Shinohara, A., Harada, M., Wakana, S., Sakaizumi, M., Han, S. H., Lin, L. K. and Kryukov, A. P. 2000. Molecular phylogeny of East Asian moles inferred from the sequence variation of the mitochondrial cytochrome $b$ gene. Genes and Genetic Systems 75: 17-24.

Yamada, M., Hosoi, E., Tamate, H. B., Nagata, J., Tatsuzawa, S., Tado, H. and Ozawa, S. 2006. Distribution of two distinct lineages of sika deer (Cervus nippon) on Shikoku island revealed by mitochondrial DNA analysis. Mammal Study 31:23-28.

Yamamoto, Y. 1986. Researches of setts of Meles meles anakuma in Mt. Nyugasa, Nagano Prefecture. Natural Environmental Science Laboratory 2: 131-139 (in Japanese with English summary).

Yamamoto, Y. 1991. Food habit of the Japanese badger (Meles meles anakuma in Mt. Nyugasa, Nagano Prefecture. Natural Environmental Science Laboratory 4: 73-83 (in Japanese with English summary). 